\title{
Exponential mixing property for automorphisms of compact Kähler manifolds
}

\author{
$\mathrm{Hao} \mathrm{Wu}$
}

\begin{abstract}
Let $f$ be a holomorphic automorphism of a compact Kähler manifold. Assume that $f$ admits a unique maximal dynamic degree $d_{p}$ with only one eigenvalue of maximal modulus. Let $\mu$ be its equilibrium measure. In this paper, we prove that $\mu$ is exponentially mixing for all d.s.h. test functions.
\end{abstract}

\section{Introduction and main results}

Let $(X, \omega)$ be a compact Kähler manifold of dimension $k$ and let $f$ be a holomorphic automorphism of $X$. Denote by $f^{*}$ the pull-back operator acting on the Hodge cohomology groups $H^{*, *}(X, \mathbb{C})$. Recall that the dynamic degree of order $q$ of $f$ is the spectral radius of $f^{*}$ on $H^{q, q}(X, \mathbb{C})$, and denoted by $d_{q}$. We have $d_{0}=d_{k}=1$. Khovanskii-Teissier-Gromov [11] proved that the function $q \mapsto \log d_{q}$ is concave. Thus there are integers $0 \leq p \leq p^{\prime} \leq k$ such that

$$
1=d_{0}<\ldots<d_{p}=\ldots=d_{p^{\prime}}>\ldots>d_{k}=1 .
$$

When $p=p^{\prime}$ and in addition, when $f^{*}$ acting on $H^{p, p}(X, \mathbb{C})$, admits only one eigenvalue of maximal modulus (necessary equal to $d_{p}$ ), there is a unique invariant probability measure $\mu:=T_{+} \wedge T_{-}$, where $T_{+}$is the Green $(p, p)$-current of $f$ and $T_{-}$ is the Green $(k-p, k-p)$-current of $f^{-1}$. They satisfy $f^{*}\left(T_{+}\right)=d_{p} T^{+}$and $f_{*}\left(T_{-}\right)=$ $d_{k-p} T_{-}$. Moreover, for any positive closed $(p, p)$-current (resp. $(k-p, k-p)$-current) $S$ of mass 1 , we have $d_{p}^{-n}\left(f^{n}\right)^{*}(S)$ (resp. $\left.d_{k-p}^{-n}\left(f^{n}\right)_{*}(S)\right)$ converge to $T_{+}\left(\operatorname{resp} . T_{-}\right)$. And $T_{+}$(resp. $T_{-}$) is the unique positive closed current in the class $\left\{T_{+}\right\}$(resp. $\left.\left\{T_{-}\right\}\right)$. The measure $\mu$ is called the equilibrium measure of $f$. For the constructions

Key words and phrases: dynamic degree, equilibrium measure, exponential mixing, superpotential.

AMS Subject Classification: $37 \mathrm{~F}, 32 \mathrm{H}$. 
of $\mu, T_{+}, T_{-}$, the readers may refer to [10]. And see e.g. [13] and [14] for interesting examples.

Recall that a function is quasi-plurisubharmonic (quasi-p.s.h. for short) on $X$ if locally it is the difference of a plurisubharmonic (p.s.h. for short) function and a smooth one. The following theorem is our first main result.

Theorem 1.1. Let $f$ be a holomorphic automorphism on a compact Kähler manifold $X$ of dimension $k$ and let $\mu$ be its equilibrium measure. Let $d_{q}$ be the dynamic degree of order $q, 0 \leq q \leq k$. Assume that there is a integer $p$ such that $d_{p}$ is strictly large than other dynamic degrees and $d_{p}$ admits only one eigenvalue of maximal modulus $d_{p}$. Then $\mu$ is exponentially mixing for bounded quasi-p.s.h. observables. More precisely, if $\delta$ is a constant such that $\max \left\{d_{p-1}, d_{p+1}\right\}<\delta<d_{p}$ and all the eigenvalues of $f^{*}$, acting on $H^{p, p}(X, \mathbb{C})$, except $d_{p}$, are strictly smaller than $\delta$. Then there exists a constant $c>0$, such that

$$
\left|\int\left(\varphi \circ f^{n}\right) \psi d \mu-\left(\int \varphi d \mu\right)\left(\int \psi d \mu\right)\right| \leq c\left(d_{p} / \delta\right)^{-n / 2}\|\varphi\|_{L^{\infty}}\|\psi\|_{L^{\infty}}
$$

for all $n \geq 0$ and all bounded quasi-p.s.h. functions $\varphi$ and $\psi$ satisfy $d d^{c} \varphi \geq-\omega, d d^{c} \psi \geq$ $-\omega$.

The conditions $d d^{c} \varphi \geq-\omega, d d^{c} \psi \geq-\omega$ in Theorem 1.1 relate to the $*$-norm defined in Section 2. Another version of Theorem 1.1 has been proved in [9] for $\varphi, \psi \in$ $\mathscr{C}^{2}$ and it can be extended to $\mathscr{C}^{\alpha}$ case, $0<\alpha \leq 2$, using interpolation theory between Banach spaces. In this case, one considers the new system $(z, w) \mapsto\left(f^{-1}(z), f(w)\right)$ on $X \times X$ and the test function $\varphi(z) \psi(w)$, which plays a linear "role" in the new system. Since $\varphi(z) \psi(w)$ is of class $\mathscr{C}^{2}$ and in particular, it is Hölder continuous, some estimates of super-potentials on the currents with Hölder continuous superpotentials imply the desired result.

However, in the study of complex dynamics, sometimes we need to investigate the behaviors of the functions with some singularities under the action of $f$. For example, the class of quasi-p.s.h. functions or d.s.h. functions (see the definition below). When $\varphi$ and $\psi$ are not of class of $\mathscr{C}^{2}$, then idea in [9] can not be directly applied. In this case, firstly, the super-potentials may not be well defined on the space of non-smooth currents. Secondly, when $\varphi$ and $\psi$ are not in $\mathscr{C}^{2}$, the function $\varphi(z) \psi(w)$ will not be Hölder continuous any more. In the proof, we do some regularization of quasi-p.s.h. functions. After that we combine the idea in [9] with some techniques in [15] to prove the main theorem. Similarly estimates of superpotentials on the currents with Hölder continuous super-potentials also are obtained at the end of Section 2.

Recall that a function $u$ on $X$ with values in $\mathbb{R} \cup\{ \pm \infty\}$ is said to be d.s.h. if outside a pluripolar set it is equal to a difference of two quasi-p.s.h. functions. Two 
d.s.h. functions are identified when they are equal out of a pluripolar set. Denote the set of d.s.h. functions by $\operatorname{DSH}(X)$. Clearly it is a vector space and equips with a norm

$$
\|u\|_{\mathrm{DSH}}:=\left|\int_{X} u \omega^{k}\right|+\min \left\|T^{ \pm}\right\|,
$$

where the minimum is taken on all positive closed $(1,1)$-currents $T^{ \pm}$such that $d d^{c} u=T^{+}-T^{-}$.

A positive measure $\nu$ on $X$ is said to be moderate if for any bounded family $\mathscr{F}$ of d.s.h. functions on $X$, there are constants $\alpha>0$ and $c>0$ such that

$$
\nu\{z \in X:|\psi(z)|>M\} \leq c e^{-\alpha M}
$$

for $M \geq 0$ and $\psi \in \mathscr{F}$ (see [5], [6] and [8]). The papers [5] and [10] show that if $f$ is a holomorphic automorphism of a compact Kähler surface or more generally, on a compact Kähler manifold, then the equilibrium measure $\mu$ of $f$ is moderate. Using the moderate property of $\mu$ and following the same approach as in the proof of [15, Theorem 1.3], we get the following theorem, which removes the boundedness conditions of $\varphi$ and $\psi$.

Theorem 1.2. Let $f, d_{p}, \mu$ be as in Theorem 1.1. Then the equilibrium measure $\mu$ is exponentially mixing for all d.s.h observables. More precisely, if $\delta$ is a constant such that $\max \left\{d_{p-1}, d_{p+1}\right\}<\delta<d_{p}$ and all the eigenvalues of $f^{*}$, acting on $H^{p, p}(X, \mathbb{C})$, except $d_{p}$, are strictly smaller than $\delta$. Then for any two d.s.h. functions $\varphi, \psi$, there exists a constant $c>0$, such that

$$
\left|\int\left(\varphi \circ f^{n}\right) \psi d \mu-\left(\int \varphi d \mu\right)\left(\int \psi d \mu\right)\right| \leq c\left(d_{p} / \delta\right)^{-n / 2}
$$

for all $n \geq 0$.

In Theorem 1.2, the constant $c$ depends on $\varphi$ and $\psi$. It is not hard to see that we can take a common $c$ for any compact family of d.s.h. functions.

Now we consider a particular case. When $X$ is a compact Kähler surface and $f$ is of positive entropy, Gromov [12] and Yomdin [16] showed that the entropy is equal to $\log d_{1}$. Thus in this case, $d_{1}>1$. Moreover, Cantat [1] proved that the eigenvalues of $f^{*}$, acting on $H^{1,1}(X, \mathbb{C})$, are $d_{1}, 1 / d_{1}$ and others with modulus 1 . Thus we get the following corollary.

Corollary 1.3. Let $f$ be a holomorphic automorphism of positive entropy on a compact Kähler surface $X$. Then the equilibrium measure $\mu$ is exponentially mixing for all d.s.h. observables. 
In this paper, the symbols $\lesssim$ and $\gtrsim$ stand for inequalities up to a multiplicative constant.

Acknowledgments. This work was supported by the grant: AcRF Tier 1 R-146-000-248-114 from National University of Singapore. I also would like to thank the referees for their remarks.

\section{Super-potentials of currents}

In this section, we will introduce the notion called super-potential. The readers may refer to [7] and [10] for details. Some estimates of super-potentials on a family of currents with Hölder continuous super-potentials are obtained at the end of this section.

Denote by $\mathscr{D}_{q}$ the real space that generated by all positive closed $(q, q)$-currents on $X$. Define a norm $\|\cdot\|_{*}$ on $\mathscr{D}_{q}$ by

$$
\|\Omega\|_{*}:=\min \left\{\left\|\Omega^{+}\right\|+\left\|\Omega^{-}\right\|\right\},
$$

where $\left\|\Omega^{ \pm}\right\|:=\left\langle\Omega^{ \pm}, \omega^{k-q}\right\rangle$ are the mass of $\Omega^{ \pm}$, and the minimum is taken over all the positive closed currents $\Omega^{ \pm}$with $\Omega=\Omega^{+}-\Omega^{-}$. Observe that $\left\|\Omega^{ \pm}\right\|$only depend on the cohomology classes of $\Omega^{ \pm}$in $H^{q, q}(X, \mathbb{R})$. We have the following lemma.

Lemma 2.1. Let $\Omega$ be a real $d d^{c}$-exact $(q, q)$-current on $X$ and assume $\Omega \geq-S$ for some positive closed $(q, q)$-current $S$, then $\|\Omega\|_{*} \leq 2\|S\|$.

Proof. Note that $\Omega+S$ is a positive closed current and we can write $\Omega$ as

$$
\Omega=(\Omega+S)-S
$$

The mass of $\Omega+S$ is $\|S\|$ because $\Omega$ is $d d^{c}$-exact.

We introduce the $*$-topology on $\mathscr{D}_{q}$ : for a sequence of currents $S_{n}$ in $\mathscr{D}_{q}$, we say $S_{n}$ converge to a current $S$ in $\mathscr{D}_{q}$ if $S_{n}$ converge to $S$ in the sense of currents and if $\left\|S_{n}\right\|_{*}$ are uniformly bounded. Note that smooth forms are dense in $\mathscr{D}_{q}$ for this topology.

Let $\mathscr{D}_{q}^{0}$ be the subspace of $\mathscr{D}_{q}$ which contains all the currents of class $\{0\}$ in $H^{q, q}(X, \mathbb{R})$. It is not hard to see $\mathscr{D}_{q}^{0}$ is closed under the above topology.

Now we define the super-potential of a current $S \in \mathscr{D}_{q}$. Fix a basis of $H^{q, q}(X, \mathbb{R})$, denoted by $\{\alpha\}:=\left\{\left\{\alpha_{1}\right\}, \ldots,\left\{\alpha_{t}\right\}\right\}$. We can take all the $\alpha_{j}$ being smooth forms. For any $R \in \mathscr{D}_{k-q+1}^{0}$, there exists a real $(k-q, k-q)$-current $U_{R}$ such that $d d^{c} U_{R}=R$. We call $U_{R}$ a potential of $R$. After adding some closed form to $U_{R}$ we can assume 
$\left\langle U_{R}, \alpha_{j}\right\rangle=0$ for all $1 \leq j \leq t$. After that we say $U_{R}$ is $\alpha$-normalized. If in addition, $R$ is smooth, then we can choose $U_{R}$ smooth.

The $\alpha$-normalized super-potential $\mathscr{U}_{S}$ of $S$ is a linear functional on the smooth forms in $\mathscr{D}_{k-q+1}^{0}$, and it is defined by

$$
\mathscr{U}_{S}(R):=\left\langle S, U_{R}\right\rangle,
$$

where $U_{R}$ is a smooth $\alpha$-normalized potential of $R$. Note that $\mathscr{U}_{S}(R)$ does not depend on the choice of $U_{R}$.

If $\mathscr{U}_{S}$ can be extended continuously to a linear functional on $\mathscr{D}_{k-q+1}^{0}$ for the *-topology we defined above, then we say $S$ has a continuous super-potential. If $S \in \mathscr{D}_{q}^{0}$, then $\mathscr{U}_{S}$ does not depend on the choice of $\alpha$. If $S$ is smooth, then it has a continuous super-potential and we have $\mathscr{U}_{S}(R)=\mathscr{U}_{R}(S)$, where $\mathscr{U}_{R}$ is the superpotential of $R$. The equality still holds if we only assume $S$ has a continuous super-potential (see [10]).

For $0<l<\infty$, we define the norm $\|\cdot\|_{\mathscr{C}^{-l}}$ and the distance $\operatorname{dist}_{l}$ on $\mathscr{D}_{q}$ by

$$
\|\Omega\|_{\mathscr{C}-l}:=\sup _{\|\Phi\|_{\mathscr{C} l} \leq 1}|\langle\Omega, \Phi\rangle| \quad \text { and } \operatorname{dist}_{l}\left(\Omega, \Omega^{\prime}\right):=\left\|\Omega-\Omega^{\prime}\right\|_{\mathscr{C}-l},
$$

where $\Phi$ is a smooth test $(k-q, k-q)$-form on $X$. For $0<l<l^{\prime}<\infty$, on any $\|$. $\|_{*}$-bounded subset of $\mathscr{D}_{p}$, we have

$$
\operatorname{dist}_{l^{\prime}} \leq \operatorname{dist}_{l} \leq c_{l, l^{\prime}}(\operatorname{dist})^{l / l^{\prime}}
$$

for some positive constant $c_{l, l^{\prime}}$ (see [10]).

For $S \in \mathscr{D}_{q}$ and constants $l>0,0<\lambda \leq 1, M \geq 0$, a super-potential $\mathscr{U}_{S}$ of $S$ is said to be $(l, \lambda, M)$-Hölder continuous if it is continuous and it satisfies

$$
\left|\mathscr{U}_{S}(R)\right| \leq M\|R\|_{\mathscr{C}-l}^{\lambda}
$$

for all $R \in \mathscr{D}_{k-q+1}^{0}$ with $\|R\|_{*} \leq 1$. If $l^{\prime}>0$ is another constant, the above identity for dist $_{l}$ and dist $l^{\prime}$ implies that $\mathscr{U}_{S}$ is also $\left(l^{\prime}, \lambda^{\prime}, M^{\prime}\right)$-Hölder continuous for some constants $\lambda^{\prime}$ and $M^{\prime}$ independent of $S$. And this definition does not depend on the normalization of the super-potential. We need the following two lemmas which are originally stated in [9].

Lemma 2.2. Let $R \in \mathscr{D}_{k-p+1}^{0}$ with $\|R\|_{*} \leq 1$ and $\mathscr{U}_{R}$ is $(2, \lambda, M)$-Hölder continuous. There is a constant $A>0$ independent of $R, \lambda$ and $M$ such that the superpotential $\mathscr{U}_{S}$ of $S$ satisfies

$$
\left|\mathscr{U}_{S}(R)\right| \leq A\left(1+\lambda^{-1} \log ^{+} M\right),
$$

for any $S \in \mathscr{D}_{p}^{0}$ with $\|S\|_{*} \leq 1$, where $\log ^{+}:=\max \{0, \log \}$. 
Lemma 2.3. Let $f, p$ be as in Theorem 1.1 and let $R \in \mathscr{D}_{k-p+1}^{0}$ whose superpotential $\mathscr{U}_{R}$ is $(2, \lambda, M)$-Hölder continuous. Then there is a constant $A_{0} \geq 1$ independent of $R, \lambda, M$ such that the super-potential $\mathscr{U}_{f_{*}(R)}$ of $f_{*}(R)$ is $\left(2, \lambda, A_{0} M\right)$-Hölder continuous.

We will use the above two lemmas to show the following result. A simple case was shown in [9, Proposition 3.1], which is crucial in the proof of exponential mixing theorem for $\mathscr{C}^{\alpha}$ observables for $0<\alpha \leq 2$. Since $T_{+}$is the unique positive current in $\left\{T_{+}\right\}$, if $S \in \mathscr{D}_{p}$, then $d_{p}^{-n}\left(f^{n}\right)^{*}(S)$ converge to a multiple of $T_{+}$.

Proposition 2.4. Let $f, d_{p}, \delta$ be as in Theorem 1.1 and $S \in \mathscr{D}_{p}$. Let $r$ be the constant such that $d_{p}\left(f^{n}\right)^{*}(S)$ converge to $r T_{+}$. Let $\left\{R_{\varepsilon}\right\}_{0<\varepsilon \leq 1 / 2}$ be a family of currents in $\mathscr{D}_{k-p+1}^{0}$ with $\left\|R_{\varepsilon}\right\|_{*} \leq 1$ whose super-potentials $\mathscr{U}_{R_{\varepsilon}}$ are $\left(2, \lambda, \varepsilon^{-2}\right)$-Hölder continuous. Let $\mathscr{U}_{n}$ and $\mathscr{U}_{+}$be the $\alpha$-normalized super-potential of $d_{p}^{-n}\left(f^{n}\right)^{*}(S)$ and $T_{+}$respectively. Then there exists a constant $A>0$ independent of the family $\left\{R_{\varepsilon}\right\}$ such that

$$
\left|\mathscr{U}_{n}\left(R_{\varepsilon}\right)-r \mathscr{U}_{+}\left(R_{\varepsilon}\right)\right| \leq-A \log \varepsilon\left(d_{p} / \delta\right)^{-n}
$$

for all $n$ and $\varepsilon$.

Proof. It was shown in [9, Section 3] and [10, Section 4] that for $S \in \mathscr{D}_{p}$ smooth and closed, we have $\left|\mathscr{U}_{n}(R)-r \mathscr{U}_{n}(R)\right| \lesssim\left(d_{p} / \delta\right)^{-n}\|R\|_{*}$ for all $R \in \mathscr{D}_{k-p+1}^{0}$. So we can subtract a smooth closed $(p, p)$-form from $S$ and assume that $S \in \mathscr{D}_{p}^{0}$ and $r=0$.

Fix a constant $\delta_{0}$ such that $\max \left\{d_{p-1}, d_{p+1}\right\}<\delta_{0}<\delta$ and $\delta_{0}$ satisfies the same properties of $\delta$ as in Theorem 1.1. By Poincaré duality, the dynamic degree $d_{p-1}$ of $f$ is equal to the dynamic degree $d_{k-p+1}\left(f^{-1}\right)$ of $f^{-1}$. Since the mass of a positive current can be computed cohomologically, we have $\left\|\left(f^{n}\right)_{*}\left(R_{\varepsilon}\right)\right\|_{*} \lesssim \delta_{0}^{n}\left\|R_{\varepsilon}\right\|_{*}$.

Define $R_{n, \varepsilon}:=c^{-1} \delta_{0}^{-n}\left(f^{n}\right)_{*}\left(R_{\varepsilon}\right)$ where $c \geq 1$ is a fixed constant large enough such that $\left\|R_{n, \varepsilon}\right\|_{*} \leq 1$ for all $n$ and $\varepsilon$. By Lemma 2.3, the super-potential of $R_{n, \varepsilon}$, denoted by $\mathscr{U}_{R_{n, \varepsilon}}$, is $\left(2, \lambda, A_{0}^{n} \varepsilon^{-2}\right)$-Hölder continuous for some $A_{0} \geq 1$. On the other hand, since $S \in \mathscr{D}_{p}^{0}$, by definition we have

$$
\mathscr{U}_{n}\left(R_{\varepsilon}\right)=d_{p}^{-n} \mathscr{U}_{S}\left(\left(f^{n}\right)_{*}\left(R_{\varepsilon}\right)\right)=c\left(d_{p} / \delta_{0}\right)^{-n} \mathscr{U}_{S}\left(R_{n, \varepsilon}\right) .
$$

Finally, applying Lemma 2.2, we obtain

$$
\begin{aligned}
\left|\mathscr{U}_{n}\left(R_{\varepsilon}\right)\right| & =c\left(d_{p} / \delta_{0}\right)^{-n}\left|\mathscr{U}_{S}\left(R_{n, \varepsilon}\right)\right| \lesssim\left(d_{p} / \delta_{0}\right)^{-n}\left(1+\lambda^{-1} \log ^{+}\left(A_{0}^{n} \varepsilon^{-2}\right)\right) \\
& \lesssim-\log \varepsilon\left(d_{p} / \delta\right)^{-n} .
\end{aligned}
$$

This finishes the proof. 


\section{Exponentially mixing of $\mu$}

From now on, let $f, d_{p}$ and $\delta$ be as in Theorem 1.1, and let $S$ be a fixed positive closed $(p, p)$-current of mass 1 on $X$. Define a sequence of currents $S_{n}$ by $S_{n}:=$ $d_{p}^{-n}\left(f^{n}\right)^{*}(S)$. We know that $S_{n}$ converge to $T_{+}$. Fix a basis $\{\alpha\}:=\left\{\left\{\alpha_{1}\right\}, \ldots,\left\{\alpha_{t}\right\}\right\}$ of $H^{p, p}(X, \mathbb{R})$. Denote by $\mathscr{U}_{n}$ and $\mathscr{U}_{+}$be the $\alpha$-normalized super-potentials of $S_{n}$ and $T_{+}$respectively.

For any bounded quasi-p.s.h. function $\phi$ on $X$ such that $d d^{c} \phi \geq-\omega,|\phi| \leq 1$, we consider the same regularization of $\phi$ as in [3, Theorem 2.1] (when $X=\mathbb{P}^{k}$, see also [7, Section 2]), which is using a standard convolution and a partition of unity to regularize the function locally, then gluing them globally by using maximal regularization function [2, I.5]. So there exists a family of smooth functions $\phi_{\varepsilon}, 0<$ $\varepsilon \leq 1 / 2$ such that $d d^{c} \phi_{\varepsilon} \geq-\omega$, and $\phi_{\varepsilon}$ decreases to $\phi_{0}:=\phi$ when $\varepsilon$ decreases to 0 . And $\phi_{\varepsilon}$ satisfies the following two estimates:

$$
\left\|\phi_{\varepsilon}-\phi\right\|_{L^{1}\left(\omega^{k}\right)} \lesssim \varepsilon \quad \text { and }\left\|\phi_{\varepsilon}\right\|_{\mathscr{E}^{2}} \lesssim \varepsilon^{-2},
$$

where the \'s are independent of $\phi$.

We define a sequence of functions $h_{n}$ and $h$ on $(0,1 / 2]$ by

$$
h_{n}(\varepsilon)=\mathscr{U}_{n}\left(d d^{c} \phi_{\varepsilon} \wedge T_{-}\right) \quad \text { and } \quad h(\varepsilon)=\mathscr{U}_{+}\left(d d^{c} \phi_{\varepsilon} \wedge T_{-}\right) .
$$

By definition,

$$
h_{n}(\varepsilon)=\left\langle S_{n} \wedge T_{-}, \phi_{\varepsilon}\right\rangle-\left\langle S_{n}, K_{\varepsilon}\right\rangle \quad \text { and } \quad h(\varepsilon)=\left\langle T_{+} \wedge T_{-}, \phi_{\varepsilon}\right\rangle-\left\langle T_{+}, K_{\varepsilon}\right\rangle,
$$

where $K_{\varepsilon}$ is a smooth closed $(k-p, k-p)$-form depends on $\varepsilon$ such that $\phi_{\varepsilon} T_{-}-K_{\varepsilon}$ is the $\alpha$-normalized potential of $d d^{c} \phi_{\varepsilon} \wedge T_{-}$, i.e. $\left\langle\phi_{\varepsilon} T_{-}-K_{\varepsilon}, \alpha_{j}\right\rangle=0$ for all $j$. Observe that $h_{n}$ converge pointwise to $h$ on $(0,1 / 2]$.

On the other hand, note that $\left\{\omega^{k}\right\}$ is a basis of $H^{k, k}(X, \mathbb{R})$. We consider the $\left\{\omega^{k}\right\}$-normalized super potential of $\mu=T_{+} \wedge T_{-}$and define the function

$$
g(\varepsilon):=\mathscr{U}_{\mu}\left(d d^{c} \phi_{\varepsilon}\right)=\left\langle T_{+} \wedge T_{-}, \phi_{\varepsilon}\right\rangle-\left\langle\omega^{k}, \phi_{\varepsilon}\right\rangle .
$$

The function $g$ is well defined at $\varepsilon=0$ because $T_{+} \wedge T_{-}$has a Hölder continuous super-potential (see [10]). We prove two lemmas first.

Lemma 3.1. There exists a constant $c>0$ independent of $\phi$ such that

$$
\left|\left\langle S_{n}, K_{\varepsilon}\right\rangle-\left\langle T_{+}, K_{\varepsilon}\right\rangle\right| \leq c\left(d_{p} / \delta\right)^{-n}
$$

for $\varepsilon \in(0,1 / 2]$. 
Proof. Let $\left(a_{n, 1}, a_{n, 2}, \ldots, a_{n, t}\right)$ be the vector which represents the class $\left\{S_{n}\right\}$ in $H^{p, p}(X, \mathbb{R})$ with respect to the basis $\{\alpha\}$, i.e. $\left\{S_{n}\right\}=\sum_{j=1}^{t} a_{n, j}\left\{\alpha_{j}\right\}$. Let $\left(b_{1}, b_{2}, \ldots\right.$, $b_{t}$ ) be the vector which represents $\left\{T_{+}\right\}$. Since $K_{\varepsilon}$ is closed, we have

$$
\left\langle S_{n}-T_{+}, K_{\varepsilon}\right\rangle=\sum_{j=1}^{t}\left\langle\left(a_{n, j}-b_{j}\right) \alpha_{j}, K_{\varepsilon}\right\rangle .
$$

Combining with $\left\langle\phi_{\varepsilon} T_{-}-K_{\varepsilon}, \alpha_{j}\right\rangle=0$ for all $j$, we get

$$
\left\langle S_{n}-T_{+}, K_{\varepsilon}\right\rangle=\sum_{j=1}^{t}\left(a_{n, j}-b_{j}\right)\left\langle\alpha_{j}, \phi_{\varepsilon} T_{-}\right\rangle .
$$

On the other hand, by Perron-Frobenius theorem, $\left\|\left\{S_{n}\right\}-\left\{T_{+}\right\}\right\| \lesssim\left(d_{p} / \delta\right)^{-n}$ in the finite dimensional vector space $H^{p, p}(X, \mathbb{R})$ (see also $[9$, Section 3$]$ ). Therefore, we have

$$
\left\|\left(a_{n, 1}-b_{1}, a_{n, 2}-b_{2}, \ldots, a_{n, t}-b_{t}\right)\right\| \lesssim\left(d_{p} / \delta\right)^{-n} .
$$

Finally, observe that $\left\langle\alpha_{j}, \phi_{\varepsilon} T_{-}\right\rangle$is uniformly bounded independent of $\phi$. Hence

$$
\left|\left\langle S_{n}-T_{+}, K_{\varepsilon}\right\rangle\right| \lesssim\left(d_{p} / \delta\right)^{-n}
$$

The proof of this lemma is finished.

Lemma 3.2. The function $g$ is Hölder continuous at 0 , more precisely, there exists a constant $c>0$ independent of $\phi$ such that for $\varepsilon \in(0,1 / 2]$, we have $\mid g(\varepsilon)-$ $g(0) \mid \leq c \varepsilon^{\alpha}$ for some $0<\alpha \leq 1$.

Proof. Since $T_{+} \wedge T_{-}$has a Hölder continuous super-potential, by definition, we have

$$
|g(\varepsilon)-g(0)| \leq M \operatorname{dist}_{2}\left(d d^{c} \phi_{\varepsilon}, d d^{c} \phi\right)^{\alpha}
$$

for some constants $0<\alpha \leq 1, M>0$.

Since $\phi_{\varepsilon}$ is decreasing when $\varepsilon$ decreases, by definition and estimates (3.1) we obtain

$$
\begin{aligned}
\operatorname{dist}_{2}\left(d d^{c} \phi_{\varepsilon}, d d^{c} \phi\right) & =\sup _{\|\Phi\|_{\mathscr{C}^{2}} \leq 1}\left|\left\langle d d^{c} \phi_{\varepsilon}-d d^{c} \phi, \Phi\right\rangle\right|=\sup _{\|\Phi\|_{\mathscr{C}^{2} \leq 1}}\left|\left\langle\phi_{\varepsilon}-\phi, d d^{c} \Phi\right\rangle\right| \\
& \lesssim\left\langle\phi_{\varepsilon}-\phi, \omega^{k}\right\rangle=\left\|\phi_{\varepsilon}-\phi\right\|_{L^{1}\left(\omega^{k}\right) \lesssim \varepsilon}
\end{aligned}
$$

since $\|\Phi\|_{\mathscr{C}^{2}} \leq 1$ implies $\pm d d^{c} \Phi \leq c^{\prime} \omega^{k}$, where $c^{\prime}$ is a positive constant only depending on $(X, \omega)$. Therefore,

$$
|g(\varepsilon)-g(0)| \leq M \operatorname{dist}_{2}\left(d d^{c} \phi_{\varepsilon}, d d^{c} \phi\right)^{\alpha} \lesssim \varepsilon^{\alpha} .
$$

The proof of this lemma is complete. 
Since $\phi_{\varepsilon}$ is smooth for every $\varepsilon \neq 0$, in particular it is Hölder continuous. We can easily obtain the estimates of $h_{n}(\varepsilon)-h(\varepsilon)$ for $\varepsilon \neq 0$ by using Proposition 2.4. Combining with the above two lemmas we get the following key proposition.

Proposition 3.3. Let $S_{n}$ and $\phi$ be as above. There exists a constant $c>0$ independent of $\phi$ such that

$$
\left\langle S_{n} \wedge T_{-}, \phi\right\rangle-\left\langle T_{+} \wedge T_{-}, \phi\right\rangle \leq c\left(d_{p} / \delta\right)^{-n}
$$

for all $n$.

Proof. Again, we fix a constant $\delta_{0}$ such that $\max \left\{d_{p-1}, d_{p+1}\right\}<\delta_{0}<\delta$ and $\delta_{0}$ satisfies the same properties of $\delta$ as in Theorem 1.1. By Lemma 2.1, $\left\|d d^{c} \phi_{\varepsilon}\right\|_{*} \leq 2$ for all $\varepsilon$, thus $\left\|d d^{c} \phi_{\varepsilon} \wedge T_{-}\right\|_{*}$ are uniformly bounded for $1<\varepsilon \leq 1 / 2$. Since $\left\|\phi_{\varepsilon}\right\|_{\mathscr{C}^{2}} \lesssim \varepsilon^{-2}$ and $T_{-}$has a Hölder continuous super-potential (see [10]), by [10, Proposition 3.4.2], $d d^{c} \phi_{\varepsilon} \wedge T_{-}$has a $\left(2, \lambda, M \varepsilon^{-2}\right)$-Hölder continuous super-potential for some constant $\lambda$ and $M$ independent of $\phi$. Multiplying $\phi$ by some constant allows us to assume $M=1$ and $\left\|d d^{c} \phi_{\varepsilon} \wedge T_{-}\right\|_{*} \leq 1$ for all $0<\varepsilon \leq 1 / 2$. Applying Proposition 2.4 to the family $\left\{d d^{c} \phi_{\varepsilon} \wedge T_{-}\right\}$instead of $\left\{R_{\varepsilon}\right\}$, we get that for $0<\varepsilon \leq 1 / 2$,

$$
h_{n}(\varepsilon)-h(\varepsilon) \lesssim-\log \varepsilon\left(d_{p} / \delta_{0}\right)^{-n}
$$

where the $\lesssim$ is independent of $\phi$. Combining this with estimates (3.1), Lemma 3.1 and Lemma 3.2 , we have for $\varepsilon \in(0,1 / 2]$,

$$
\begin{aligned}
& \left\langle S_{n} \wedge T_{-}, \phi\right\rangle-\left\langle T_{+} \wedge T_{-}, \phi\right\rangle \leq\left\langle S_{n} \wedge T_{-}, \phi_{\varepsilon}\right\rangle-\left\langle T_{+} \wedge T_{-}, \phi\right\rangle \\
& =\left\langle S_{n} \wedge T_{-}, \phi_{\varepsilon}\right\rangle-\left\langle T_{+} \wedge T_{-}, \phi_{\varepsilon}\right\rangle+\left\langle T_{+} \wedge T_{-}, \phi_{\varepsilon}\right\rangle-\left\langle T_{+} \wedge T_{-}, \phi\right\rangle \\
& =h_{n}(\varepsilon)-h(\varepsilon)+\left\langle S_{n}, K_{\varepsilon}\right\rangle-\left\langle T_{+}, K_{\varepsilon}\right\rangle+g(\varepsilon)+\left\langle\omega^{k}, \phi_{\varepsilon}\right\rangle-g(0)-\left\langle\omega^{k}, \phi\right\rangle \\
& \lesssim-\log \varepsilon\left(d_{p} / \delta_{0}\right)^{-n}+\left(d_{p} / \delta_{0}\right)^{-n}+\varepsilon^{\alpha}+\varepsilon,
\end{aligned}
$$

where the first inequality is because $\phi_{\varepsilon}$ is decreasing as $\varepsilon$ decreasing and $S_{n} \wedge T_{-}$is positive.

Finally, since $\alpha \leq 1$, by taking $\varepsilon=\left(d_{p} / \delta_{0}\right)^{-n / \alpha}$, we get

$$
\left\langle S_{n} \wedge T_{-}, \phi\right\rangle-\left\langle T_{+} \wedge T_{-}, \phi\right\rangle \lesssim n \log \left(d_{p} / \delta_{0}\right)\left(d_{p} / \delta_{0}\right)^{-n}+\left(d_{p} / \delta_{0}\right)^{-n} \lesssim\left(d_{p} / \delta\right)^{-n}
$$

Since the constant $c$ in Lemma 3.1 and Lemma 3.2 are independent of $\phi$, the $\lesssim$ above is independent of $\phi$.

In Proposition 3.3, the constant $c$ depends on $S$. Note that we do not have a lower bound estimate. Otherwise, we can follow the approach in [9] to show Theorem 1.1 directly. Here we need some extra techniques from [15]. 
Proof of Theorem 1.1. Multiplying $\varphi$ and $\psi$ by some constant allows us to assume $\|\varphi\|_{L^{\infty}} \leq 1 / 2$ and $\|\psi\|_{L^{\infty}} \leq 1 / 2$. It is sufficient to prove Theorem 1.1 for $n$ even because applying it to $\varphi$ and $\psi \circ f$ gives the case of odd $n$. Using the invariance of $\mu$, it is enough to show that

$$
\left|\left\langle\mu,\left(\varphi \circ f^{n}\right)\left(\psi \circ f^{-n}\right)\right\rangle-\langle\mu, \varphi\rangle\langle\mu, \psi\rangle\right| \leq c\left(d_{p} / \delta\right)^{-n}
$$

for some $c>0$. It is equivalent to prove

$$
\left\langle\mu,\left(\varphi \circ f^{n}\right)\left(\psi \circ f^{-n}\right)\right\rangle-\langle\mu, \varphi\rangle\langle\mu, \psi\rangle \leq c\left(d_{p} / \delta\right)^{-n}
$$

and

$$
\left\langle\mu,\left(\varphi \circ f^{n}\right)\left(-\psi \circ f^{-n}\right)\right\rangle-\langle\mu, \varphi\rangle\langle\mu,-\psi\rangle \leq c\left(d_{p} / \delta\right)^{-n} .
$$

For $j=1,2$, we define

$$
\varphi_{j}^{+}:=\varphi^{2}+j \varphi+A, \quad \varphi_{j}^{-}:=\varphi^{2}+j \varphi-A, \quad \psi_{j}^{+}:=\psi^{2}+j \psi+A, \quad \psi_{j}^{-}:=-\psi^{2}-j \psi+A,
$$

where $A$ is a positive constant whose value will be determined later. Consider the following eight functions on $X \times X$ :

$$
\Phi_{j l}^{+}(z, w):=\varphi_{j}^{+}(z) \psi_{l}^{+}(w), \quad \Phi_{j l}^{-}(z, w):=\varphi_{j}^{-}(z) \psi_{l}^{-}(w),
$$

where $j, l=1,2$. We need the following lemma.

Lemma 3.4. The functions $\Phi_{j l}^{ \pm}$are quasi-p.s.h. on $X \times X$ for A large enough.

Proof. We only show $\Phi_{11}^{+}$and $\Phi_{11}^{-}$are quasi-p.s.h. because the other cases can be obtained in the same way. By a direct computation (see also [15, Lemma 3.1]), we have

$$
\begin{aligned}
i \partial \bar{\partial} \Phi_{11}^{+}= & \left(\psi^{2}+\psi+A\right)((2 \varphi+1) i \partial \bar{\partial} \varphi+2 i \partial \varphi \wedge \bar{\partial} \varphi)+(2 \varphi+1)(2 \psi+1) i \partial \varphi \wedge \bar{\partial} \psi \\
& +(2 \varphi+1)(2 \psi+1) i \partial \psi \wedge \bar{\partial} \varphi+\left(\varphi^{2}+\varphi+A\right)((2 \psi+1) i \partial \bar{\partial} \psi+2 i \partial \psi \wedge \bar{\partial} \psi) .
\end{aligned}
$$

Combining with the identity

$$
i \partial \varphi \wedge \bar{\partial} \varphi+i \partial \varphi \wedge \bar{\partial} \psi+i \partial \psi \wedge \bar{\partial} \varphi+i \partial \psi \wedge \bar{\partial} \psi=i \partial(\varphi+\psi) \wedge \bar{\partial}(\varphi+\psi) \geq 0,
$$

we get

$$
\begin{aligned}
i \partial \bar{\partial} \Phi_{11}^{+} \geq & \left(\psi^{2}+\psi+A\right)(2 \varphi+1) i \partial \bar{\partial} \varphi+\left(\varphi^{2}+\varphi+A\right)(2 \psi+1) i \partial \bar{\partial} \psi \\
& +\left(2 \psi^{2}+2 \psi+2 A-(2 \varphi+1)(2 \psi+1)\right) i \partial \varphi \wedge \bar{\partial} \varphi \\
& +\left(2 \varphi^{2}+2 \varphi+2 A-(2 \varphi+1)(2 \psi+1)\right) i \partial \psi \wedge \bar{\partial} \psi .
\end{aligned}
$$


Recall that we assume $\|\varphi\|_{L^{\infty}} \leq 1 / 2$ and $\|\psi\|_{L^{\infty}} \leq 1 / 2$. So $2 \varphi+1 \geq 0,2 \psi+1 \geq 0$. We take $A$ large enough such that $\psi^{2}+\psi+A, \varphi^{2}+\varphi+A, 2 \psi^{2}+2 \psi+2 A-(2 \varphi+1)(2 \psi+$ 1), $2 \varphi^{2}+2 \varphi+2 A-(2 \varphi+1)(2 \psi+1)$ are all positive. Since $\varphi$ and $\psi$ are quasi-p.s.h. on $\mathrm{X}$ and $i \partial \varphi \wedge \bar{\partial} \varphi, i \partial \psi \wedge \bar{\partial} \psi$ are positive, we deduce that $\Phi_{11}^{+}$is quasi-p.s.h. on $X \times X$.

For $\Phi_{11}^{-}$, we have

$$
\begin{aligned}
i \partial \bar{\partial} \Phi_{11}^{-}= & \left(-\psi^{2}-\psi+A\right)((2 \varphi+1) i \partial \bar{\partial} \varphi+2 i \partial \varphi \wedge \bar{\partial} \varphi)-(2 \varphi+1)(2 \psi+1) i \partial \varphi \wedge \bar{\partial} \psi \\
& -(2 \varphi+1)(2 \psi+1) i \partial \psi \wedge \bar{\partial} \varphi+\left(\varphi^{2}+\varphi-A\right)(-(2 \psi+1) i \partial \bar{\partial} \psi-2 i \partial \psi \wedge \bar{\partial} \psi) .
\end{aligned}
$$

Combining with the identity

$$
i \partial \varphi \wedge \bar{\partial} \varphi-i \partial \varphi \wedge \bar{\partial} \psi-i \partial \psi \wedge \bar{\partial} \varphi+i \partial \psi \wedge \bar{\partial} \psi=i \partial(\varphi-\psi) \wedge \bar{\partial}(\varphi-\psi) \geq 0
$$

we get

$$
\begin{aligned}
i \partial \bar{\partial} \Phi_{11}^{-} \geq & \left(-\psi^{2}-\psi+A\right)(2 \varphi+1) i \partial \bar{\partial} \varphi+\left(-\varphi^{2}-\varphi+A\right)(2 \psi+1) i \partial \bar{\partial} \psi \\
& +\left(-2 \psi^{2}-2 \psi+2 A-(2 \varphi+1)(2 \psi+1)\right) i \partial \varphi \wedge \bar{\partial} \varphi \\
& +\left(-2 \varphi^{2}-2 \varphi+2 A-(2 \varphi+1)(2 \psi+1)\right) i \partial \psi \wedge \bar{\partial} \psi
\end{aligned}
$$

Repeating the same argument as above, we get that $\Phi_{11}^{-}$is quasi-p.s.h. for $A$ large enough. The proof is complete.

We choose $A$ large enough such that all the $\Phi_{j l}^{ \pm}$are bounded and quasi-p.s.h. on $X \times X$. Note that the choice of $A$ is independent of $\varphi$ and $\psi$. Define $\widetilde{\omega}:=\pi_{1}^{*} \omega+\pi_{2}^{*} \omega$, where $\pi_{1}, \pi_{2}$ are the two canonical projections of $X \times X$ onto its factors. Then $\widetilde{\omega}$ is the canonical Kähler form on $X \times X$. Recall that we assume $d d^{c} \varphi \geq-\omega, d d^{c} \psi \geq-\omega$. From the computations in Lemma 3.4, we deduce that $d d^{c} \Phi_{11}^{+} \geq-3 A \widetilde{\omega}$ when $A$ is large. And observe that $\Phi_{11}^{+}$is bounded by $4 A^{2}$.

Next we consider the automorphism $F$ of $X \times X$ which is defined by

$$
F(z, w):=\left(f^{-1}(z), f(w)\right) .
$$

By using Künneth formula, one can show that the dynamic degree of order $k$ of $F$ is equal to $d_{p}^{2}$ (see also $[9$, Section 4$]$ ), and the dynamical degrees and the eigenvalues of $F^{*}$ on $H^{k, k}(X \times X, \mathbb{R})$, except $d_{p}^{2}$, are strictly smaller than $d_{p} \delta$. Hence $F$ and $d_{p} \delta$ satisfy the conditions of $f$ and $\delta$ respectively in Theorem 1.1.

It is not hard to see that the Green $(k, k)$-currents of $F$ and $F^{-1}$ are $T_{-} \otimes T_{+}$ and $T_{+} \otimes T_{-}$respectively, and they satisfy

$$
F^{*}\left(T_{-} \otimes T_{+}\right)=d_{p}^{2}\left(T_{-} \otimes T_{+}\right), F_{*}\left(T_{+} \otimes T_{-}\right)=d_{p}^{2}\left(T_{+} \otimes T_{-}\right) .
$$

In particular, they have Hölder continuous super-potentials. Let $\Delta$ denote the diagonal of $X \times X$. Then $[\Delta]$ is a positive closed $(k, k)$-current on $X \times X$. With the help of $F$, we get the following estimates. 
Lemma 3.5. There exists a constant $c>0$ such that

$$
\left\langle\mu,\left(\varphi_{j}^{+} \circ f^{n}\right)\left(\psi_{l}^{+} \circ f^{-n}\right)\right\rangle-\left\langle\mu, \varphi_{j}^{+}\right\rangle\left\langle\mu, \psi_{l}^{+}\right\rangle \leq c\left(d_{p} / \delta\right)^{-n}
$$

and

$$
\left\langle\mu,\left(\varphi_{j}^{-} \circ f^{n}\right)\left(\psi_{l}^{-} \circ f^{-n}\right)\right\rangle-\left\langle\mu, \varphi_{j}^{-}\right\rangle\left\langle\mu, \psi_{l}^{-}\right\rangle \leq c\left(d_{p} / \delta\right)^{-n}
$$

for all $j, l$ and $n$.

Proof. We only show this lemma holds for $\varphi_{1}^{+}$and $\psi_{1}^{+}$, the proofs of others are similar. For the automorphism $F$, consider the sequence of currents $d_{p}^{-2 n}\left(F^{n}\right)^{*}[\Delta]$, which are positive closed currents of mass 1 converging to $T_{-} \otimes T_{+}$. Since $d d^{c} \Phi_{11}^{+} \geq$ $-3 A \widetilde{\omega}$ and $\left|\Phi_{11}^{+}\right| \leq 4 A^{2}$, after dividing $\Phi_{11}^{+}$by $4 A^{2}$, we can assume $d d^{c} \Phi_{11}^{+} \geq-\widetilde{\omega}$ and $\left|\Phi_{11}^{+}\right| \leq 1$. Applying Proposition 3.3 to $d_{p}^{-2 n}\left(F^{n}\right)^{*}[\Delta], T_{+} \otimes T_{-}$and $\Phi_{11}^{+}$instead of $S_{n}, T_{-}$and $\phi$, we deduce that there exists a constant $c>0$ such that

$$
\left\langle d_{p}^{-2 n}\left(F^{n}\right)^{*}[\Delta] \wedge\left(T_{+} \otimes T_{-}\right), \Phi_{11}^{+}\right\rangle-\left\langle\left(T_{-} \otimes T_{+}\right) \wedge\left(T_{+} \otimes T_{-}\right), \Phi_{11}^{+}\right\rangle \leq c\left(d_{p}^{2} /\left(d_{p} \delta\right)\right)^{-n}
$$

for all $n$. Here $c$ is independent of $\varphi$ and $\psi$ because $A$ is independent of them.

On the other hand, by definition, we have

$$
\begin{aligned}
\left\langle d_{p}^{-2 n}\left(F^{n}\right)^{*}[\Delta] \wedge\left(T_{+} \otimes T_{-}\right), \Phi_{11}^{+}\right\rangle & =\left\langle[\Delta], d_{p}^{-2 n}\left(F^{n}\right)_{*}\left[\left(T_{+} \otimes T_{-}\right) \wedge \Phi_{11}^{+}\right]\right\rangle \\
& =\left\langle[\Delta] \wedge\left(T_{+} \otimes T_{-}\right), \Phi_{11}^{+} \circ F^{-n}\right\rangle \\
& =\left\langle T_{+} \wedge T_{-},\left(\varphi_{1}^{+} \circ f^{n}\right)\left(\psi_{1}^{+} \circ f^{-n}\right)\right\rangle,
\end{aligned}
$$

and

$$
\left\langle\left(T_{-} \otimes T_{+}\right) \wedge\left(T_{+} \otimes T_{-}\right), \Phi_{11}^{+}\right\rangle=\left\langle\mu \otimes \mu, \Phi_{11}^{+}\right\rangle=\left\langle\mu, \varphi_{1}^{+}\right\rangle\left\langle\mu, \psi_{1}^{+}\right\rangle .
$$

This finishes the proof of this lemma.

Now we can finish the proof of Theorem 1.1 using the invariant property of $\mu$.

End of the proof of Theorem 1.1. Consider $\alpha_{11}^{+}=2, \alpha_{22}^{+}=\alpha_{11}^{-}=\alpha_{21}^{-}=\alpha_{12}^{-}=1$ and $\alpha_{21}^{+}=\alpha_{12}^{+}=\alpha_{22}^{-}=0$. A direct computation gives

$$
\begin{aligned}
& \sum_{j, l=1,2}\left(\alpha_{j l}^{+}\left(\varphi_{j}^{+} \circ f^{n}\right)\left(\psi_{l}^{+} \circ f^{-n}\right)+\alpha_{j l}^{-}\left(\varphi_{j}^{-} \circ f^{n}\right)\left(\psi_{l}^{-} \circ f^{-n}\right)\right) \\
& \quad=\left(\varphi \circ f^{n}\right)\left(\psi \circ f^{-n}\right)+\beta_{1} \varphi^{2} \circ f^{n}+\beta_{2} \psi^{2} \circ f^{-n}+\beta_{3} \varphi \circ f^{n}+\beta_{4} \psi \circ f^{-n}+\beta_{5}
\end{aligned}
$$

for some constants $\beta_{t}, 1 \leq t \leq 5$. We now apply this identity and Lemma 3.5. Observe that the invariance of $\mu$ implies that

$$
\left\langle\mu, \varphi^{m} \circ f^{ \pm n}\right\rangle=\left\langle\mu, \varphi^{m}\right\rangle \quad \text { and } \quad\left\langle\mu, \psi^{m} \circ f^{ \pm n}\right\rangle=\left\langle\mu, \psi^{m}\right\rangle .
$$


Hence the terms involving $\beta_{t}$ cancel each other out. We obtain

$$
\left\langle\mu,\left(\varphi \circ f^{n}\right)\left(\psi \circ f^{-n}\right)\right\rangle-\langle\mu, \varphi\rangle\langle\mu, \psi\rangle \leq\left(\sum_{j, l=1,2}\left(\alpha_{j l}^{+}+\alpha_{j l}^{-}\right)\right) c\left(d_{p} / \delta\right)^{-n}=6 c\left(d_{p} / \delta\right)^{-n}
$$

Similarly, taking $\gamma_{11}^{-}=2, \gamma_{11}^{+}=\gamma_{21}^{+}=\gamma_{12}^{+}=\gamma_{22}^{-}=1$ and $\gamma_{22}^{+}=\gamma_{21}^{-}=\gamma_{12}^{-}=0$, we get

$$
\left\langle\mu,\left(\varphi \circ f^{n}\right)\left(-\psi \circ f^{-n}\right)\right\rangle-\langle\mu, \varphi\rangle\langle\mu,-\psi\rangle \leq\left(\sum_{j, l=1,2}\left(\gamma_{j l}^{+}+\gamma_{j l}^{-}\right)\right) c\left(d_{p} / \delta\right)^{-n}=6 c\left(d_{p} / \delta\right)^{-n} \text {. }
$$

The above two inequalities imply inequality (3.2) and finish the proof of Theorem 1.1 .

Using the moderate property of $\mu$ and the technical of replacing $\delta$ by $\delta_{0}$, we can prove Theorem 1.2.

Proof of Theorem 1.2. It is enough to prove this theorem for all negative quasip.s.h. functions $\varphi$ and $\psi$. Multiplying them by some constant allows us to assume $d d^{c} \varphi \geq-\omega, d d^{c} \psi \geq-\omega$ and $\langle\mu,|\varphi|\rangle \leq 1,\langle\mu,|\psi|\rangle \leq 1$. Define

$$
\varphi_{1}:=\max \{\varphi,-M\}, \quad \psi_{1}:=\max \{\psi,-M\},
$$

and

$$
\varphi_{2}:=\varphi-\varphi_{1}, \quad \psi_{2}:=\psi-\psi_{1} .
$$

Then $\varphi_{1}$ and $\psi_{1}$ are bounded quasi-p.s.h. functions which satisfy $d d^{c} \varphi_{1} \geq$ $-\omega, d d^{c} \psi_{1} \geq-\omega$. Fix a constant $\delta_{0}$ such that $\max \left\{d_{p-1}, d_{p+1}\right\}<\delta_{0}<\delta$ and $\delta_{0}$ satisfies the same properties of $\delta$ as in Theorem 1.1. Applying Theorem 1.1 to $\varphi_{1}$ and $\psi_{1}$, we get

$$
\left|\int\left(\varphi_{1} \circ f^{n}\right) \psi_{1} d \mu-\left(\int \varphi_{1} d \mu\right)\left(\int \psi_{1} d \mu\right)\right| \lesssim\left(d_{p} / \delta_{0}\right)^{-n / 2} M^{2} .
$$

On the other hand, since $\mu$ is moderate, by [4, Lemma 2.1] or the proof of [15, Theorem 1.3], we get for some $\alpha>0$,

$$
\begin{aligned}
&\left\|\varphi_{2}\right\|_{L^{1}(\mu)} \lesssim e^{-\alpha M / 2},\left\|\psi_{2}\right\|_{L^{1}(\mu)} \lesssim e^{-\alpha M / 2}, \\
&\left\|\varphi_{2}\right\|_{L^{2}(\mu)} \lesssim e^{-\alpha M / 2}, \quad\left\|\psi_{2}\right\|_{L^{2}(\mu)} \lesssim e^{-\alpha M / 2} .
\end{aligned}
$$

From the invariance of $\mu$, we have that $\left\|\varphi_{2} \circ f^{n}\right\|_{L^{p}(\mu)}=\left\|\varphi_{2}\right\|_{L^{p}(\mu)}$ and $\| \psi_{2} \circ$ $f^{n}\left\|_{L^{p}(\mu)}=\right\| \psi_{2} \|_{L^{p}(\mu)}$ for $1 \leq p \leq \infty$. We do the following direct computation (see also [15, Theorem 1.3]),

$$
\begin{aligned}
& \left|\left\langle\mu,\left(\varphi \circ f^{n}\right) \psi\right\rangle-\langle\mu, \varphi\rangle\langle\mu, \psi\rangle\right| \\
& =\left|\left\langle\mu,\left(\varphi_{1} \circ f^{n}+\varphi_{2} \circ f^{n}\right)\left(\psi_{1}+\psi_{2}\right)\right\rangle-\left\langle\mu, \varphi_{1}+\varphi_{2}\right\rangle\left\langle\mu, \psi_{1}+\psi_{2}\right\rangle\right|
\end{aligned}
$$




$$
\begin{aligned}
\leq & \left|\left\langle\mu,\left(\varphi_{1} \circ f^{n}\right) \psi_{1}\right\rangle-\left\langle\mu, \varphi_{1}\right\rangle\left\langle\mu, \psi_{1}\right\rangle\right|+\left|\left\langle\mu,\left(\varphi_{1} \circ f^{n}\right) \psi_{2}\right\rangle\right|+\left|\left\langle\mu,\left(\varphi_{2} \circ f^{n}\right) \psi_{1}\right\rangle\right| \\
& +\left|\left\langle\mu,\left(\varphi_{2} \circ f^{n}\right) \psi_{2}\right\rangle\right|+\left|\left\langle\mu, \varphi_{2}\right\rangle\left\langle\mu, \psi_{1}\right\rangle\right|+\left|\left\langle\mu, \varphi_{1}\right\rangle\left\langle\mu, \psi_{2}\right\rangle\right|+\left|\left\langle\mu, \varphi_{2}\right\rangle\left\langle\mu, \psi_{2}\right\rangle\right| \\
\leq & \left|\left\langle\mu,\left(\varphi_{1} \circ f^{n}\right) \psi_{1}\right\rangle-\left\langle\mu, \varphi_{1}\right\rangle\left\langle\mu, \psi_{1}\right\rangle\right|+M\left\|\varphi_{2}\right\|_{L^{1}(\mu)}+M\left\|\psi_{2}\right\|_{L^{1}(\mu)} \\
& +\left\|\varphi_{2}\right\|_{L^{2}(\mu)}\left\|\psi_{2}\right\|_{L^{2}(\mu)}+\left\|\varphi_{2}\right\|_{L^{1}(\mu)}+\left\|\psi_{2}\right\|_{L^{1}(\mu)}+\left\|\varphi_{2}\right\|_{L^{1}(\mu)}\left\|\psi_{2}\right\|_{L^{1}(\mu)} \\
\lesssim & \left(d_{p} / \delta_{0}\right)^{-n / 2} M^{2}+(2 M+2) e^{-\alpha M / 2}+2 e^{-\alpha M} .
\end{aligned}
$$

Taking $M=\left(n \log \left(d_{p} / \delta_{0}\right)\right) / \alpha$, we obtain the estimate

$$
\left(d_{p} / \delta_{0}\right)^{-n / 2} M^{2}+(2 M+2) e^{-\alpha M / 2}+2 e^{-\alpha M} \lesssim n^{2}\left(d_{p} / \delta_{0}\right)^{-n / 2} \lesssim\left(d_{p} / \delta\right)^{-n / 2} .
$$

Therefore,

$$
\left|\int\left(\varphi \circ f^{n}\right) \psi d \mu-\left(\int \varphi d \mu\right)\left(\int \psi d \mu\right)\right| \lesssim\left(d_{p} / \delta\right)^{-n / 2} .
$$

The proof is finished.

Remark 3.6. In the last step of the proof above, there is an $n^{2}$ appearing in the middle before replacing $\delta_{0}$ by $\delta$. It somehow represents the singularities of $\varphi$ and $\psi$. The constant $c$ in Theorem 1.1 and Theorem 1.2 can be made more explicit, but it needs a long calculation so we chose not to do here.

\section{References}

1. Cantat, S., Dynamique des automorphismes des surfaces K3, Acta Math. 187 (2001), $1-57$.

2. Demailly, J.-P., Complex Analytic and Differential Geometry. http:// ww-fourier.ujf-grenoble.fr/ demailly/

3. Dinh, T.-C., MA, X. and NGuYÊn, V.-A., Equidistribution speed for Fekete points associated with an ample line bundle, Ann. Sci. Éc. Norm. Supér. (4) 50 (2017), 545-578.

4. Dinh, T.-C. and NGuYÊN, V.-A., Characterization of Monge-Ampère measures with Hölder continuous potentials, J. Funct. Anal. 266 (2014), 67-84.

5. Dinh, T.-C., NGuyên, V.-A. and Sibony, N., Exponential estimates for plurisubharmonic functions and stochastic dynamics, J. Differ. Geom. 84 (2010), 465-488.

6. Dinh, T.-C. and Sibony, N., Dynamique des applications d'allure polynomiale, J. Math. Pures Appl. (9) 82 (2003), 367-423.

7. Dinh, T.-C. and Sibony, N., Super-potentials of positive closed currents, intersection theory and dynamics, Acta Math. 203 (2009), 1-82.

8. Dinh, T.-C. and Sibony, N., Dynamics in several complex variables: endomorphisms of projective spaces and polynomial-like mappings, in Holomorphic dynamical systems, Lecture Notes in Math. 1998, pp. 165-294, Springer, Berlin, 2010. 
9. Dinh, T.-C. and Sibony, N., Exponential mixing for automorphisms on compact Kähler manifolds, in Dynamical numbers-interplay between dynamical systems and number theory, Contemp. Math. 532, pp. 107-114, Am. Math. Soc., Providence, 2010.

10. Dinh, T.-C. and Sibony, N., Super-potentials for currents on compact Kähler manifolds and dynamics of automorphisms, J. Algebraic Geom. 19 (2010), 473-529.

11. Gromov, M., Convex sets and Kähler manifolds, in Advances in differential geometry and topology, pp. 1-38, World Sci. Publ., Teaneck, NJ, 1990.

12. Gromov, M., On the entropy of holomorphic maps, Enseign. Math. 49 (2003), 217235.

13. Oguiso, K., Free automorphisms of positive entropy on smooth Kähler surfaces, in Algebraic geometry in east Asia-Taipei 2011, Adv. Stud. Pure Math. 65, pp. 187-199, Math. Soc. Japan, Tokyo, 2015.

14. Oguiso, K. and Truong, T. T., Explicit examples of rational and Calabi-Yau threefolds with primitive automorphisms of positive entropy, J. Math. Sci. Univ. Tokyo 22 (2015), 361-385.

15. Wu, H., Exponential mixing property for Hénon-Sibony maps of $\mathbb{C}^{k}$, Preprint, 2019. arXiv : 1910.02437.

16. Yomdin, Y., Volume growth and entropy, Isr. J. Math. 57 (1987), 285-300.

\section{Hao $\mathrm{Wu}$}

Department of Mathematics

National University of Singapore

10, Lower Kent Ridge Road

Singapore SG-119076

Singapore

e0011551@u.nus.edu

Received June 3, 2020

in revised form October 2, 2020 